\title{
On Certain Topological Structures of Product Normed Space Valued Paranormed Space of Summable Sequences
}

\author{
J.K.Srivastava* and Narayan Prasad Pahari** \\ * Department of Mathematics and Statistics \\ Gorakhpur University, India \\ ${ }^{* *}$ Central Department of Mathematic \\ Tribhuvan University, Kirtipur, Kathmandu, Nepal \\ Email: nppahari@gmail.com
}

\begin{abstract}
The aim of this paper is to introduce and study a new vector valued sequence space $S\left(\left(Z,\|(., .)\|_{Z}\right), \bar{\gamma}, \bar{u}\right)$ with terms from a product normed space as a generalization of sequence space studied by Srivastava and Pahari (2011) which is itself the generalization of the familiar absolutely summable sequence space $l$. We investigate its linear structure with respect to the co-ordinate wise vector operation and explore the conditions in terms of different $\bar{u}$ and $\bar{\gamma}$ so that a class is contained in another class of same kind and thereby derive the conditions of their equality. Finally we investigate the paranormed structure of $S\left(\left(Z,\|(., .)\|_{Z}\right), \bar{\gamma}, \bar{u}\right)$ by endowing it with a suitable natural paranorm.
\end{abstract}

Key words: Sequence space, paranormed space, product normed space, GK- sequence space.

\section{INTRODUCTION}

The notion of vector valued sequence space is a generalized form of spaces of scalar valued sequences, and its terms consist of sequences from a vector space. Let $A$ be a normed space over $\boldsymbol{C}$, the field of complex numbers and let $\omega(A)$ denotes the linear space of all $A$ valued sequences with usual coordinate-wise operations. We shall denote $\omega$ $(\boldsymbol{C})$ by $\boldsymbol{\omega}$. Any subspace of $\boldsymbol{\omega}$ is then called a sequence space. The various types of vector valued sequence spaces has been significantly developed by several workers for instances, Köthe (1970), Maddox (1980), Kamthan and Gupta (1980), Ruckle (1981), Malkowski and Rakocevic (2004), Khan (2008), Kolk (2011), Srivastava and Pahari (2011, 2012 \& 2013).

The notion of paranormed space is closely related to linear metric space (Wilansky, 1978). A paranormed space $(A, \Phi)$ is a linear space $A$ with zero element $\theta$, together with a function $\Phi: A \rightarrow \boldsymbol{R}_{+}$(called a paranorm on $A$ ) which satisfies the following axioms:

PN1: $\Phi(\theta)=0$;

PN2: $\Phi(\xi)=\Phi(-\xi)$, for all $\xi \in A$;

PN3: $\Phi(\xi+\eta) \leq \Phi(\xi)+\Phi(\eta)$,for all $\xi, \eta \in A$;

and PN4: Scalar multiplication is continuous

i.e., if $\left\langle\gamma_{n}>\right.$ is a sequence of scalars with $\gamma_{n} \rightarrow \gamma$ as

$n \rightarrow \infty$ and $<\xi_{n}>$ is a sequence of vectors with $\Phi\left(\xi_{n}-\xi\right)$

$\rightarrow 0$ as $n \rightarrow \infty$, then $\Phi\left(\gamma_{n} \xi_{n}-\gamma \xi\right) \rightarrow 0$ as $n \rightarrow \infty$.

Note that the continuity of scalar multiplication is equivalent to

(i) if $\Phi\left(\xi_{n}\right) \rightarrow 0$ and $\gamma_{n} \rightarrow \gamma$ as $n \rightarrow \infty$, then

$\Phi\left(\gamma_{n} \xi_{n}\right) \rightarrow 0$ as $n \rightarrow \infty$; and (ii) if $\gamma_{n} \rightarrow 0$ as $n \rightarrow \infty$ and $\xi$ be any element in $A$, then $\Phi\left(\gamma_{n} \xi\right) \rightarrow 0$ (Wilansky, 1978).

A paranorm $\Phi$ is called total if $\Phi(\xi)=0$ implies $\xi=\theta$ (Wilansky, 1978). The studies of paranorm on sequence spaces were initiated by Maddox (1969). Various types of paranorms on the spaces of sequences and functions were further studied in the works of Parashar and Choudhary (1994), Bhardwaj and Bala (2007), Khan (2008), Tiwari and Srivastava (2008 \& 2010), Pahari (2011 \& 2013), Srivastava and Pahari $(2011,2012 \& 2013)$.

Let $\left(A,\|.\|_{A}\right)$ and $\left(B,\|.\|_{B}\right)$ be Banach spaces over the field $C$ of complex numbers. Clearly the linear space structure of $A$ and $B$ provides the Cartesian product of $A$ and $B$ given by

$Z=A \times B=\{(\xi, \eta): \xi \in A, \eta \in B\}$ forms a normed linear space over $\boldsymbol{C}$ under the algebraic operations $\alpha\left(\xi_{1}, \eta_{1}\right)+\beta\left(\xi_{2}, \eta_{2}\right)=\left(\alpha \xi_{1}+\beta \xi_{2}, \alpha \eta_{1}+\beta \eta_{2}\right)$ with the $\operatorname{norm}\|(\xi, \eta)\|_{A \times B}=\max \left\{\|\xi\|_{A},\|\eta\|_{B}\right\}$

where $\left(\xi_{1}, \eta_{1}\right),\left(\xi_{2}, \eta_{2}\right),(\xi, \eta) \in Z$ and $\alpha, \beta \in \boldsymbol{C}$.

Moreover, since $\left(A,\|.\|_{A}\right)$ and $\left(B,\|.\|_{B}\right)$ are Banach spaces therefore the Cartesian product $\left(Z,\|(., .)\|_{Z}\right)$ is also a Banach space.

In studying various classes of a scalar valued sequence space (see, Kamthan and Gupta (1980), we have the following definition:

A normed space $A$ - valued topological sequence space $V(A)$ equipped with the linear topology $\mathfrak{I}$ is said to be a GK-space if the projection map $\pi_{k}: V(A) \rightarrow A$ defined by $\pi_{k}(\bar{\xi})=\xi_{k}$ is continuous for each $k$.

Let $\bar{u}=\left(u_{k}\right)$ and $\bar{v}=\left(v_{k}\right)$ be any sequences of strictly positive real numbers and $\bar{\gamma}=\left(\gamma_{k}\right)$ and $\bar{\mu}=\left(\mu_{k}\right)$ be sequences of non-zero complex numbers. We now 
introduce the following class of Banach space $Z$ - valued sequences:

$$
\begin{gathered}
S\left(\left(Z,\|(., .)\|_{Z}\right), \bar{\gamma}, \bar{u}\right)=\left\{\bar{u}=<\left(\xi_{k}, \eta_{k}\right)>:\right. \\
\left.\left(\xi_{k}, \eta_{k}\right) \in Z, \sum_{k=1}^{\infty}\left\|\gamma_{k}\left(\xi_{k}, \eta_{k}\right)\right\|^{u_{k}}<\infty\right\} . \ldots \ldots \ldots . .
\end{gathered}
$$

Further, when $\gamma_{k}=1$ for all $k$, then $S\left(\left(Z,\|(., .)\|_{Z}\right), \bar{\gamma}, \bar{u}\right)$ will be denoted by $S\left(\left(Z,\|(., .)\|_{Z}\right), \bar{u}\right)$ and when $u_{k}=1$ for all $k$; then $S\left(\left(Z,\|(., .)\|_{Z}\right), \bar{\gamma}, \bar{u}\right)$ will be denoted by $S\left(\left(Z,\|(., .)\|_{Z}\right), \bar{\gamma}\right)$.

Several properties of bilinear vector valued sequence spaces defined on product normed spaces have been introduced and examined by the various workers , see Sanchezl et al. (2000), Castillo et al. (2001), Yilmaz and Solak (2004), which generalize and unify a number of existing sequence spaces and function spaces.

\section{RESULTS}

In this section we show the linearity of the class

$S\left(\left(Z,\|(., .)\|_{Z}\right), \bar{\gamma}, \bar{u}\right)$ with respect to co-ordinate wise vector operations and then explore the conditions on $\bar{u}$ and $\bar{\gamma}$ so that a class $S\left(\left(Z,\|(., .)\|_{Z}\right), \bar{\gamma}, \bar{u}\right)$ is contained in or equal to another similar class. Finally we investigate the paranormed structures of $S\left(\left(Z,\|(., .)\|_{Z}\right), \bar{\gamma}, \bar{u}\right)$ by endowing it with a suitable natural paranorm.

As far the as linear space structure of the class $S\left(\left(Z,\|(., .)\|_{Z}\right), \bar{\gamma}, \bar{u}\right)$ over the field $\mathbf{C}$ are concerned, we shall take point-wise vector operations, i.e. for

$\bar{s}=\left\langle\left(\xi_{k}, \eta_{k}\right)>, \bar{z}=\left\langle\left(\xi_{k}^{\prime}, \eta_{k}^{\prime}\right)>\right.\right.$

$\in S\left(\left(Z,\|(., .)\|_{Z}\right), \bar{\gamma}, \bar{u}\right)$ and $\alpha, \beta \in C$, we have

$$
\alpha \bar{s}+\beta \bar{z}=\alpha<\left(\xi_{k}, \eta_{k}\right)>+\beta<\left(\xi_{k}^{\prime}, \eta_{k}^{\prime}\right)>
$$$$
=<\left(\alpha \xi_{k}+\beta \xi_{k}^{\prime}, \alpha \eta_{k}+\beta \eta_{k}^{\prime}\right)>\text {. }
$$

The zero element of $S\left(\left(Z,\|(., .)\|_{Z}\right), \bar{\gamma}, \bar{u}\right)$ will be denoted by $\bar{\theta}=<(0,0),(0,0),(0,0), \ldots>$. Throughout the work, we shall denote $r_{k}=\left|\frac{\gamma_{k}}{\mu_{k}}\right|^{u_{k}}$.

Moreover, we write $M$ for $\max \left(1, \sup _{k} u_{k}\right)$. But when the sequences $\left\langle u_{k}\right\rangle$ and $\left\langle v_{k}\right\rangle$ occur, then to distinguish $M$ we use the notations $M(u)$ and $M(v)$ respectively.

Theorem 1: If $\sup _{k} u_{k}$ is finite, then $S\left(\left(Z,\|(., .)\|_{Z}\right), \bar{\gamma}, \bar{u}\right)$ forms a linear space over $\boldsymbol{C}$.

Proof:

Assume that $\sup _{k} u_{k}<\infty$ and $\bar{s}=<\left(\xi_{k}, \eta_{k}\right)>$ and $\bar{z}=<\left(\xi_{k}^{\prime}, \eta_{k}^{\prime}\right)>\in S\left(\left(Z,\|(., .)\|_{Z}\right), \bar{\gamma}, \bar{u}\right)$.

So that $\sum_{k=1}^{\infty}\left\|\gamma_{k}\left(\xi_{k}, \eta_{k}\right)\right\|^{u_{k}}<\infty$ and $\sum_{k=1}^{\infty}\left\|\gamma_{k}\left(\xi_{k}^{\prime}, \eta_{k}^{\prime}\right)\right\|^{u_{k}}<\infty$.

Now, considering $\left\|\gamma_{k}\left(\left(\xi_{k}, \eta_{k}\right)+\left(\xi_{k}^{\prime}, \eta_{u_{k} / M}^{\prime}\right)\right)\right\|^{u_{k} / M}$
$\leq\left\|\gamma_{k}\left(\xi_{k}, \eta_{k}\right)\right\|^{k^{\prime}}+\left\|\gamma_{k}\left(\xi_{k}^{\prime}, \eta_{k}^{\prime}\right)\right\|^{k^{\prime}}$

and therefore

$$
\begin{aligned}
& \sum_{k=1}^{\infty}\left\|\gamma_{k}\left(\left(\xi_{k}, \eta_{k}\right)+\left(\xi_{k}^{\prime}, \eta_{k}^{\prime}\right)\right)\right\|^{u_{k} / M} \\
& \leq \sum_{k=1}^{\infty}\left\|\gamma_{k}\left(\xi_{k}, \eta_{k}\right)\right\|^{u_{k} / M}+\sum_{k=1}^{\infty}\left\|\gamma_{k}\left(\xi_{k}^{\prime}, \eta_{k}^{\prime}\right)\right\|^{u_{k} / M}<\infty
\end{aligned}
$$

and hence $\bar{s}+\bar{z} \in S\left(\left(Z,\|(., .)\|_{Z}\right), \bar{\gamma}, \bar{u}\right)$.

Similarly for any scalar $\alpha$,

$$
\begin{aligned}
\left\|\alpha \gamma_{k}\left(\xi_{k}, \eta_{k}\right)\right\|^{u_{k} / M} & =|\alpha|^{u_{k / M}}\left\|\gamma_{k}\left(\xi_{k}, \eta_{k}\right)\right\|^{u_{k} / M} \\
& \leq \max (1,|\alpha|)\left\|\gamma_{k}\left(\xi_{k}, \eta_{k}\right)\right\|^{u_{k} / M}
\end{aligned}
$$

and therefore

$$
\begin{aligned}
& \sum_{k=1}^{\infty}\left\|\alpha \gamma_{k}\left(\xi_{k}, \eta_{k}\right)\right\|^{u_{k} / M} \\
& =\sum_{k=1}^{\infty}|\alpha|^{u_{k} / M}\left\|\gamma_{k}\left(\xi_{k}, \eta_{k}\right)\right\|^{u_{k} / M} \\
& \leq \max (1,|\alpha|) \sum_{k=1}^{\infty}\left\|\gamma_{k}\left(\xi_{k}, \eta_{k}\right)\right\|^{u_{k} / M}<\infty
\end{aligned}
$$

and shows that $\alpha \bar{s} \in S\left(\left(Z,\|(., .)\|_{Z}\right), \bar{\gamma}, \bar{u}\right)$. This implies that $S\left(\left(Z,\|(., .)\|_{Z}\right), \bar{\gamma}, \bar{u}\right)$ forms a linear space over $\boldsymbol{C}$.

Theorem 2: If $S\left(\left(Z,\|(., .)\|_{Z}\right), \bar{\gamma}, \bar{u}\right)$ forms a linear space over $C$,then $\sup _{k} u_{k}$ is finite.

Proof:

Suppose that $S\left(\left(Z,\|(., .)\|_{Z}\right), \bar{\gamma}, \bar{u}\right)$ forms a linear space over $\mathbf{C}$ but $\sup _{k} u_{k}=\infty$. Then there exists a sequence $<$ $k(n)>$ of positive integers satisfying $1 \leq k(n)<k(n+1)$, $n \geq 1$ for which

$u_{k(n)}>n$,for each $n \geq 1$.

Now taking $(\xi, \eta) \in Z$ with $\|(\xi, \eta)\|=1$,

we define a sequence $\bar{s}=<\left(\xi_{k}, \eta_{k}\right)>$ where $\left(\xi_{k}, \eta_{k}\right)$ is given by

$$
\left(\xi_{k}, \eta_{k}\right)=\gamma_{k(n)}^{-1} n^{-2 / u_{k(n)}}(\xi, \eta), \text { for } k=k(n), n \geq 1
$$$$
=\langle 0,0\rangle \text {, otherwise }
$$

Then we have

$$
\begin{aligned}
& \sum_{k=1}^{\infty}\left\|\gamma_{k}\left(\xi_{k}, \eta_{k}\right)\right\|^{u_{k}}=\sum_{n=1}^{\infty}\left\|\gamma_{k(n)}\left(\xi_{k(n)}, \eta_{k(n)}\right)\right\|^{u_{k(n)}} \\
& \quad \sum_{n=1}^{\infty}\left\|n^{-2 / u_{k(n)}}(\xi, \eta)\right\|^{u_{k(n)}}=\sum_{n=1}^{\infty} \frac{1}{n^{2}}<\infty .
\end{aligned}
$$


Thus we see that $\bar{s} \in S\left(\left(Z,\|(., .)\|_{Z}\right), \bar{\gamma}, \bar{u}\right)$ but on the other hand for $k=k(n), n \geq 1$ and in view of (1) and (2) for the scalar $\alpha=2$, we have

$$
\begin{aligned}
\sum_{k=1}^{\infty}\left\|\gamma_{k}\left[\alpha\left(\xi_{k}, \eta_{k}\right)\right]\right\|^{u_{k}} & =\sum_{n=1}^{\infty}\left\|\gamma_{k(n)}\left[2\left(\xi_{k(n)}, \eta_{k(n)}\right)\right]\right\|^{u_{k(n)}} \\
& =\sum_{n=1}^{\infty}|2|^{u_{k(n)}}\left\|n^{-1 / u_{k(n)}}(\xi, \eta)\right\|^{u_{k(n)}} \\
& =\sum_{n=1}^{\infty} 2^{u_{k(n)}} \cdot \frac{1}{n}>\sum_{n=1}^{\infty} \frac{2^{n}}{n} \geq 1
\end{aligned}
$$

This shows that $\alpha \bar{s} \notin S\left(\left(Z,\|(., .)\|_{Z}\right), \bar{\gamma}, \bar{u}\right)$, a contradiction. This completes the proof of the theorem.

Combining Theorem 1 and Theorem 2, we get:

Theorem 3: $S\left(\left(Z,\|(., .)\|_{Z}\right), \bar{\gamma}, \bar{u}\right)$ forms a linear space over $C$ if and only if $\sup _{k} u_{k}$ is finite.

Theorem 4: For any $\bar{u}=\left\langle u_{k}\right\rangle$ and if $\left\langle r_{k}\right\rangle$ has finite limit superior, then

$S\left(\left(Z,\|(., .)\|_{Z}\right), \bar{\mu}, \bar{u}\right) \subseteq S\left(\left(Z,\|(., .)\|_{Z}\right), \bar{\gamma}, \bar{u}\right)$

Proof :

Assume that $\lim \sup _{k} r_{k}<\infty$. Then there exists a constant $L>0$ such that $\left|\gamma_{k}\right|^{u_{k}}<L\left|\mu_{k}\right|^{u_{k}}$ for all large values of $k$. or, $\left\|\gamma_{k}<\xi_{k}, \eta_{k}>\right\|^{u_{k}}<L\left\|\mu_{k}\left(\xi_{k}, \eta_{k}\right)\right\|^{u_{k}}$.

Let $\bar{s}=<\left(\xi_{k}, \eta_{k}\right)>\in S\left(\left(Z,\|(., .)\|_{Z}\right), \bar{\mu}, \bar{u}\right)$,

so that $\sum_{k=1}^{\infty}\left\|\mu_{k}\left(\xi_{k}, \eta_{k}\right)\right\|^{u_{k}}<\infty$.

Now, $\sum_{k=1}^{\infty}\left\|\gamma_{k}\left(\xi_{k}, \eta_{k}\right)\right\|^{u_{k}}<L \sum_{k=1}^{\infty}\left\|\mu_{k}\left(\xi_{k}, \eta_{k}\right)\right\|^{u_{k}}<\infty$

and hence $\bar{s} \in S\left(\left(Z,\|(., .)\|_{Z}\right), \bar{\gamma}, \bar{u}\right)$.

This clearly implies that

$S\left(\left(Z,\|(., .)\|_{Z}\right), \bar{\mu}, \bar{u}\right) \subseteq S\left(\left(Z,\|(., .)\|_{Z}\right), \bar{\gamma}, \bar{u}\right)$.This completes the proof.

Theorem 5: For any $\bar{u}=\left\langle u_{k}\right\rangle$, if

$S\left(\left(Z,\|(., .)\|_{Z}\right), \bar{\mu}, \bar{u}\right) \subseteq S\left(\left(Z,\|(., .)\|_{Z}\right), \bar{\gamma}, \bar{u}\right)$ then $\left\langle r_{k}\right\rangle$ has finite limit superior.

Proof :

Suppose that the inclusion

$S\left(\left(Z,\|(., .)\|_{Z}\right), \bar{\mu}, \bar{u}\right) \subseteq S\left(\left(Z,\|(., .)\|_{Z}\right), \bar{\gamma}, \bar{u}\right)$

holds but lim $\sup _{k} r_{k}=\infty$. Then we can find a sequence

$<k(n)>$ of positive integers with $k(n)<k(n+1), n \geq 1$ for which

$\left|\gamma_{k(n)}\right|^{u_{k(n)}}>n\left|\mu_{k(n)}\right|^{u_{k(n)}}$

Corresponding to $(\xi, \eta) \in Z$ with $\|(\xi, \eta)\|=1$,

we define the sequence $\bar{s}=\left\langle\left(\xi_{k}, \eta_{k}\right)>\right.$ where $\left(\xi_{k}, \eta_{k}\right)=\mu_{k(n)}^{-1} n^{-2 / u_{k(n)}}(\xi, \eta)$, for $k=k(n), n \geq 1$

$=\langle 0,0\rangle$, otherwise

Then we have

$$
\begin{aligned}
\sum_{k=1}^{\infty}\left\|\mu_{k}\left(\xi_{k}, \eta_{k}\right)\right\|^{u_{k}} & =\sum_{n=1}^{\infty}\left\|\mu_{k(n)}\left(\xi_{k(n)}, \eta_{k(n)}\right)\right\|^{u_{k(n)}} \\
& =\sum_{n=1}^{\infty} \frac{1}{n^{2}}<\infty
\end{aligned}
$$

and so $\bar{s}=\left\langle\left(\xi_{k}, \eta_{k}\right)>\right.$ is in $S\left(\left(Z,\|(., .)\|_{Z}\right), \bar{\mu}, \bar{u}\right)$. But on the other hand in view of (3) and (4), we have

$$
\begin{aligned}
\sum_{k=1}^{\infty}\left\|\gamma_{k}\left(\xi_{k}, \eta_{k}\right)\right\|^{u_{k}} & =\sum_{n=1}^{\infty}\left\|\gamma_{k(n)}\left(\xi_{k(n)}, \eta_{k(n)}\right)\right\|^{u_{k(n)}} \\
& \geq \sum_{n=1}^{\infty} n\left|\mu_{k(n)}\right|^{u_{k(n)}} \|\left(\xi_{k(n)}, \eta_{k(n)} \|^{u_{k(n)}}\right. \\
& =\sum_{n=1}^{\infty} \frac{1}{n}=\infty \text { and therefore }
\end{aligned}
$$

$\bar{s}=\left\langle\left(\xi_{k}, \eta_{k}\right)\right\rangle \notin S\left(\left(Z,\|(., .)\|_{Z}\right), \bar{\gamma}, \bar{u}\right)$, a contradiction. This completes the proof of the theorem.

If Theorems 4 and 5 are combined, we get

Theorem 6: For any $\bar{u}=\left\langle u_{k}\right\rangle$,

$S\left(\left(Z,\|(., .)\|_{Z}\right), \bar{\mu}, \bar{u}\right) \subseteq S\left(\left(Z,\|(., .)\|_{Z}\right), \bar{\gamma}, \bar{u}\right)$

if and only if $\left\langle r_{k}\right\rangle$ has finite limit superior.

Theorem 7: For any $\bar{u}=\left\langle u_{k}\right\rangle$ and

$S\left(\left(Z,\|(., .)\|_{Z}\right), \bar{\gamma}, \bar{u}\right) \subseteq S\left(\left(Z,\|(., .)\|_{Z}\right), \bar{\mu}, \bar{u}\right)$ then $\left\langle r_{k}\right\rangle$ has positive limit inferior.

Proof :

Assume that $\lim \inf _{k} r_{k}>0$. Then there exists a constant $m>0$ such that $m\left|\mu_{k}\right|^{u_{k}}<\left|\gamma_{k}\right|^{u_{k}}$ for all large values of $k$.

or, $m\left\|\mu_{k}\left(\xi_{k}, \eta_{k}\right)\right\|^{u_{k}} \leq\left\|\gamma_{k}\left(\xi_{k}, \eta_{k}\right)\right\|^{u_{k}}$.

Let $\bar{s}=<\left(\xi_{k}, \eta_{k}\right)>\in S\left(\left(Z,\|(., .)\|_{Z}\right), \bar{\gamma}, \bar{u}\right)$,

so that $\sum_{k=1}^{\infty}\left\|\gamma_{k}\left(\xi_{k}, \eta_{k}\right)\right\|^{u_{k}}<\infty$. Now,

$\sum_{k=1}^{\infty}\left\|\mu_{k}\left(\xi_{k}, \eta_{k}\right)\right\|^{u_{k}}<\frac{1}{m} \sum_{k=1}^{\infty}\left\|\gamma_{k}\left(\xi_{k}, \eta_{k}\right)\right\|^{u_{k}}<\infty$ and hence

$\bar{s}=<\left(\xi_{k}, \eta_{k}\right)>\in S\left(\left(Z,\|(., .)\|_{Z}\right), \bar{\mu}, \bar{u}\right)$.

This clearly implies that

$S\left(\left(Z,\|(., .)\|_{Z}\right), \bar{\gamma}, \bar{u}\right) \subseteq S\left(\left(Z,\|(., .)\|_{Z}\right), \bar{\mu}, \bar{u}\right)$.

The proof is now complete.

Theorem 8: For any $\bar{u}=\left\langle u_{k}\right\rangle$,

$S\left(\left(Z,\|(., .)\|_{Z}\right), \bar{\gamma}, \bar{u}\right) \subseteq S\left(\left(Z,\|(., .)\|_{Z}\right), \bar{\mu}, \bar{u}\right)$

then $\left\langle r_{k}\right\rangle$ has positive limit inferior. 
Proof : Let the inclusion,

$S\left(\left(Z,\|(., .)\|_{Z}\right), \bar{\gamma}, \bar{u}\right) \subseteq S\left(\left(Z,\|(., .)\|_{Z}\right), \bar{\mu}, \bar{u}\right)$ hold but $\lim \inf _{k} r_{k}=0$. Then we can find a sequence $\langle k(n)\rangle$ of positive integers with $k(n)<k(n+1), n \geq 1$ such that for each $n \geq 1$

$n\left|\gamma_{k(n)}\right|^{u_{k(n)}}<\left|\mu_{k(n)}\right|^{u_{k(n}}$

Corresponding to $(\xi, \eta) \in Z$ with $\|(\xi, \eta)\|=1$, we define a sequence $\bar{s}=\left\langle\left(\xi_{k}, \eta_{k}\right)\right\rangle$, where

$$
\begin{aligned}
& \left(\xi_{k}, \eta_{k}\right)=\gamma_{k(n)}^{-1} n^{-2 / u_{k(n)}}(\xi, \eta), \text { for } k=k(n), n \geq 1 \\
& =(0,0) \text {, otherwise. }
\end{aligned}
$$

Then we have $\sum_{k=1}^{\infty}\left\|\gamma_{k}\left(\xi_{k}, \eta_{k}\right)\right\|^{u_{k}}$

$$
=\sum_{n=1}^{\infty}\left\|\gamma_{k(n)}\left(\xi_{k(n)}, \eta_{k(n)}\right)\right\|^{u_{k(n)}}=\sum_{n=1}^{\infty} \frac{1}{n^{2}}<\infty
$$

and so $\bar{s}=<\left(\xi_{k}, \eta_{k}\right)>$ is in $S\left(\left(Z,\|(., .)\|_{Z}\right), \bar{\gamma}, \bar{u}\right)$.

But on the other hand in view of (5) and (6) we have

$$
\begin{aligned}
\sum_{k=1}^{\infty}\left\|\mu_{k}\left(\xi_{k}, \eta_{k}\right)\right\|^{u_{k}} & =\sum_{n=1}^{\infty}\left\|\mu_{k(n)}\left(\xi_{k(n)}, \eta_{k(n)}\right)\right\|^{u_{k(n)}} \\
& =\sum_{n=1}^{\infty}\left|\frac{\mu_{k(n)}}{\gamma_{k(n)}}\right|^{u_{k(n)}} \cdot \frac{1}{n^{2}}>\sum_{n=1}^{\infty} \frac{1}{n}=\infty
\end{aligned}
$$

and therefore $\bar{s}=<\left(\xi_{k}, \eta_{k}\right)>\notin S\left(\left(Z,\|(., .)\|_{Z}\right), \bar{\mu}, \bar{u}\right)$, which is a contradiction. This completes the proof. If Theorems 7 and 8 are combined, we get

Theorem 9: For any $\bar{u}=<u_{k}>$,

$S\left(\left(Z,\|(., .)\|_{Z}\right), \bar{\gamma}, \bar{u}\right) \subseteq S\left(\left(Z,\|(., .)\|_{Z}\right), \bar{\mu}, \bar{u}\right)$ if and only if $\left\langle r_{k}\right\rangle$ has positive limit inferior.

If Theorems 6 and 9 are combined, we get

Theorem 10: For any $\bar{u}=\left\langle u_{k}\right\rangle$,

$S\left(\left(Z,\|(., .)\|_{Z}\right), \bar{\gamma}, \bar{u}\right)=S\left(\left(Z,\|(., .)\|_{Z}\right), \bar{\mu}, \bar{u}\right)$ if and only if $0<\liminf \operatorname{in}_{k} \leq \lim \sup _{k} r_{k}<\infty$.

Corollary 11: For any $\bar{u}=<u_{k}>$,

(i) $S\left(\left(Z,\|(., .)\|_{Z}\right), \bar{\gamma}, \bar{u}\right) \subseteq S\left(\left(Z,\|(., .)\|_{Z}\right), \bar{u}\right)$ if and only if $\liminf _{k}\left|\gamma_{k}\right|^{u_{k}}>0$;

(ii) $S\left(\left(Z,\|(., .)\|_{Z}\right), \bar{u}\right) \subseteq S\left(\left(Z,\|(., .)\|_{Z}\right), \bar{\gamma}, \bar{u}\right)$ if and only if $\lim \sup _{k}\left|\gamma_{k}\right|^{u_{k}}<\infty$;

(iii) $S\left(\left(Z,\|(., .)\|_{Z}\right), \bar{\gamma}, \bar{u}\right)=S\left(\left(Z,\|(., .)\|_{Z}\right), \bar{u}\right)$ if and only if $0<\liminf _{k}\left|\gamma_{k}\right|^{u_{k}} \leq \lim \sup _{k}\left|\gamma_{k}\right|^{u_{k}}<\infty$. Proof:

By taking $\mu_{k}=1$ for all $k$, in Theorems 6,9 and 10 , the assertions (i),(ii) \& (iii) follow.

Theorem 12: For any sequence $\bar{\gamma}=\left\langle\gamma_{k}\right\rangle$, if $u_{k} \leq v_{k}$ for all but finitely many $k$, then
$S\left(\left(Z,\|(., .)\|_{Z}\right), \bar{\gamma}, \bar{u}\right) \subseteq S\left(\left(Z,\|(., .)\|_{Z}\right), \bar{\gamma}, \bar{v}\right)$. Proof:

Let $\bar{s}=<\left(\xi_{k}, \eta_{k}\right)>\in S\left(\left(Z,\|(., .)\|_{Z}\right), \bar{\gamma}, \bar{u}\right)$,

then clearly $\sum_{k=1}^{\infty}\left\|\gamma_{k}\left(\xi_{k}, \eta_{k}\right)\right\|^{u_{k}}<\infty$.

This shows that there exists $K \geq 1$ such that $\left\|\gamma_{k}\left(\xi_{k}, \eta_{k}\right)\right\|<1$, for all $k \geq K$.

Thus $\left\|\gamma_{k}\left(\xi_{k}, \eta_{k}\right)\right\|^{v_{k}} \leq\left\|\gamma_{k}\left(\xi_{k}, \eta_{k}\right)\right\|^{u_{k}}$ for all $k \geq K$. This clearly shows that

$\sum_{k=1}^{\infty}\left\|\gamma_{k}\left(\xi_{k}, \eta_{k}\right)\right\|^{v_{k}}<\infty$ and hence $\bar{s}=<\left(\xi_{k}, \eta_{k}\right)>$ $\in S\left(\left(Z,\|(., .)\|_{Z}\right), \bar{\gamma}, \bar{v}\right)$. This completes the proof.

Combining Theorem 9 and Theorem 12, we get:

Theorem 13: For any $\bar{\gamma}=<\gamma_{k}>, \bar{\mu}=<\mu_{k}>, \bar{u}=<u_{k}>$ and $\bar{v}=\left\langle v_{k}\right\rangle$, if

(i) $\lim \inf _{k} r_{k}>0$; and

(ii) $u_{k} \leq v_{k}$, for all but finitely many $k$, hold together, then

$S\left(\left(Z,\|(., .)\|_{Z}\right), \bar{\gamma}, \bar{u}\right) \subseteq S\left(\left(Z,\|(., .)\|_{Z}\right), \bar{\mu}, \bar{v}\right)$.

Let $\sup _{k} u_{k}<\infty$. For $\bar{s} \in S\left(\left(Z,\|(., .)\|_{Z}\right), \bar{\gamma}, \bar{u}\right)$.

We now define

$\Phi_{\bar{\gamma}, \bar{u}}(\bar{s})=\left(\sum_{k=1}^{\infty}\left\|\gamma_{k}\left(\xi_{k}, \eta_{k}\right)\right\|^{u_{k}}\right)^{1 / M}$

In the following Theorems, $\Phi$ will denote $\Phi \bar{\gamma}, \bar{u}$.

Theorem 14: Let $\sup _{k} \quad u_{k}<\infty$ and $A, B$ be the normed spaces with $Z=A \times B$, then $S\left(\left(Z,\|(., .)\|_{Z}\right), \bar{\gamma}, \bar{u}\right)$ forms a total paranormed - space with respect to $\Phi$ Proof:

For $\bar{s}, \bar{t} \in S\left(\left(Z,\|(., .)\|_{Z}\right), \bar{\gamma}, \bar{u}\right)$,we can easily verify that $\Phi$ defined by (7) satisfy the following axioms:

PN1: $\Phi(\bar{s}) \geq 0$, and $\Phi(\bar{s})=0$ if and only if $\bar{s}=\bar{\theta}$,

$\mathrm{PN} 2: \Phi(\bar{s}+\bar{t}) \leq \Phi(\bar{u})+\Phi(\bar{t})$,

PN3: $\Phi(\alpha \bar{s}) \leq A(\alpha) \Phi(\bar{s})$, where $\alpha \in C$.

Finally to prove the continuity of scalar multiplication i.e. PN4, it suffices to show that

(a) $\Phi\left(\bar{s}^{(n)}\right) \rightarrow 0$ and $\alpha_{n} \rightarrow \alpha$ imply

$$
\Phi\left(\alpha_{n} \bar{s}^{(n)}\right) \rightarrow 0 ; \text { and }
$$

(b) $\alpha_{n} \rightarrow 0$ implies $\Phi\left(\alpha_{n} \bar{s}\right) \rightarrow 0$ for each

$\bar{s} \in S\left(\left(Z,\|(., .)\|_{Z}\right), \bar{\gamma}, \bar{u}\right)$.

Suppose $\left|\alpha_{n}\right| \leq L$ for all $n \geq 1$, then we get

$\Phi\left(\alpha_{n} \bar{s}^{(n)}\right) \leq \sup _{k}\left|\alpha_{n}\right|^{{ }_{k} / M}\left(\sum_{k=1}^{\infty}\left\|\gamma_{k}\left(\xi_{k}^{(n)}, \eta_{k}^{(n)}\right)\right\|^{\left.{ }^{k}\right)^{1 / M}}\right.$ 
$\leq \max (1,|L|) \Phi\left(\bar{s}^{(n)}\right)$ and so (a) follows.

For (b), suppose that $\bar{s} \in S\left(\left(Z,\|(., .)\|_{Z}\right), \bar{\gamma}, \bar{u}\right)$ and $\alpha_{n} \rightarrow$ 0 . Then for $\varepsilon>0$ there exist positive integers $K$ and $N$ such that

$\sum_{k=K}^{\infty}\left\|\gamma_{k}\left(\xi_{k}, \eta_{k}\right)\right\|^{u_{k}}<\left(\frac{\varepsilon}{2}\right)^{M}$ and when $n \geq N$
$\sum_{k=1}^{K-1}\left|\alpha_{n}\right|^{u_{k}}\left\|\gamma_{k}\left(\xi_{k}, \eta_{k}\right)\right\|^{u_{k}}<\left(\frac{\varepsilon}{2}\right)^{M}$ and $\left|\alpha_{n}\right| \leq 1$.

Thus for all $n \geq N$ we see that

$\Phi\left(\alpha_{n} \bar{s}\right) \leq\left(\sum_{k=1}^{K-1}\left\|\alpha_{n} \gamma_{k}\left(\xi_{k}, \eta_{k}\right)\right\|^{u_{k}}\right)^{1 / M}$

$+\left(\sum_{k=n}^{\infty}\left\|\gamma_{k}\left(\xi_{k}, \eta_{k}\right)\right\|^{u_{k}}\right)^{1 / M}<\varepsilon$, and hence (b) follows.

Theorem 15: Let $\sup _{k} u_{k}<\infty$, then if $A$ and $B$ are normed spaces with $Z=A \times B$ then

$S\left(\left(Z,\|(., .)\|_{Z}\right), \bar{\gamma}, \bar{u}\right)$ is a GK-space with respect to $\xi$. Proof:

For each $k \geq 1$, the continuity of

$\pi_{k}: S\left(\left(Z,\|(., .)\|_{Z}\right), \bar{\gamma}, \bar{u}\right) \rightarrow Z$,

where $\pi_{k}(\bar{s})=\left(\xi_{k}, \eta_{k}\right)$,follows from the inequality

$\pi_{k}(\bar{s})=\left\|\left(\xi_{k}, \eta_{k}\right)\right\| \leq\left|\gamma_{k}\right|^{-1}(\xi(\bar{s}))^{M / u_{k}}$.

Thus $S\left(\left(Z,\|(., .)\|_{Z}\right), \bar{\gamma}, \bar{u}\right)$ is a GK-space.

\section{CONCLUSION}

In this paper, we have examined the linear topological structure by endowing a suitable natural paranorm of a new vector valued sequence space $S\left(\left(Z,\|(., .)\|_{Z}\right), \bar{\gamma}, \bar{u}\right)$ with terms from a product normed space. In fact, these results can be used for further generalization to investigate topological properties of other absolutely summable sequence and function spaces.

\section{REFERENCES}

Bhardwaj,V.N. and Bala, I. 2007. Banach space valued sequence space $\ell_{M}(X, p)$. Int. J. of Pure and Appl. Maths. 41(5): 617-626.

Castillo, J., Garcia, R. and Jaramillo, J. 2001. Extension of Bilinear Forms on Banach Spaces. Proceedings of the American Mathematical Society. 129(12): 3647 3656.

Kamthan, P.K. and Gupta, M. 1980. Sequence and series , lecture notes. 65 Marcel Dekker Inc.

Khan, V.A. 2008 . On a new sequence space defined by Orlicz functions. Common Fac. Sci. Univ. Ank-series 57( 2) : 25-33

Kolk,E. 2011. Topologies in generalized Orlicz sequence spaces. Filomat 25(4): 191-211.

Köthe, G. 1970. Topological vector spaces. Springer Verlag, Berlin Heidelberg, New York.
Maddox, I. J. 1969. Some properties of paranormed sequence spaces. London. J. Math. Soc. 2(1): 316322.

Maddox, I. J. 1980. Infinite matrices of operators. Lecture Notes in Mathematics 786. Springer- Verlag Berlin, Heidelberg, New York.

Malkowski, E. and Rakocevic, V. 2004. An introduction into the theory of sequence spaces and measures of non-compactness.

Pahari, N.P. 2011. On Banach Space Valued Sequence Space $l_{\infty}(X, M, \quad \bar{\lambda}, \bar{p}, L)$ Defined by Orlicz Function.Nepal Jour. of Science and Tech. 12: 252259.

Pahari,N.P. 2013. On Certain Topological Structures of Paranormed Orlicz Space $(S((X,|I| I),. \Phi, \bar{\alpha}, \bar{u}), F)$ of Vector Valued Sequences, International Jour. of Mathematical Archive. 4 (11): 231-241.

Parashar, S.D. and Choudhary, B. 1994. Sequence spaces defined by Orlicz functions; Indian J. Pure Appl. Maths. 25(4) : 419-428.

Ruckle. W.H. 1981. Sequence spaces. Pitman Advanced Publishing Programme.

Sanchez1, F., Garcia1, R. and Villanueva, I. 2000. Extension of Multilinear Operators on Banach Spaces. Extracta Mathematica.15 (2) : 291 - 334.

Srivastava, J. K. and Pahari, N.P. 2011. On Banach space valued sequence space $l_{M}(X, \bar{\lambda}, \bar{p}, L)$ defined by Orlicz function. South East Asian J.Math. \& Math. Sc.. 10(1): 39-49.

Srivastava,J.K. and Pahari, N. P. 2011. On 2- normed space valued sequence space $l_{M}(X,\|.,\|,. \quad \lambda, \bar{p})$ defined by Orlicz function. Proc. of Indian Soc. of Math. and Mathematical Sciences 6 : 243-251.

Srivastava,J.K. and Pahari, N. P. 2012 . On vector valued paranormed sequence space $c_{0}(X, M, \bar{\lambda}, \bar{p})$ defined by Orlicz function. J. Rajasthan Acad. of Phy. Sci. 11(2) :11-24.

Srivastava, J.K. and Pahari,N.P. 2013. On 2-Banach space valued paranormed sequence space $c_{0}(X, M, \| ., . I, \bar{\lambda}, \bar{p})$ defined by Orlicz function. Jour. of Rajasthan Academy of Physical Sc. 12(3): 319-338.

Tiwari, R. K and Srivastava, J. K. 2008. On certain Banach space valued function spaces- I. Math. Forum. 20: 14-31.

Tiwari, R. K and Srivastava, J. K. 2010. On certain Banach space valued function spaces- II. Math. Forum. 22: 1-14.

Yilmaz, Y. and Solak,I. 2004. Operator Perfectness and Normality of Vector-Valued Sequence Spaces. Thai Journal of Mathematics 2 (2) : 247-257.

Wilansky, A. 1978. Modern methods in topological vector spaces. McGraw-Hill Book Co. Inc. New York. 